\title{
Clinical application of kampo medicine (rikkunshito) for common and/or intractable symptoms of the gastrointestinal tract
}

\author{
Kazunari Tominaga * and Tetsuo Arakawa \\ Department of Gastroenterology, Osaka City University Graduate School of Medicine, Osaka, Japan
}

\section{Edited by:}

Akio Inui, Kagoshima University Graduate School of Medical and Dental Sciences, Japan

\section{Reviewed by:}

Wayne B. Lau, Thomas Jefferson University, USA

Xiao Y. Tian, Houston Methodist

Research Institute, USA

*Correspondence:

Kazunari Tominaga, Department of Gastroenterology, Osaka City University Graduate School of Medicine, 1-4-3, Asahimachi,

Abeno-ku, Osaka 545-8585, Japan e-mail: tomy@med.osaka-cu.ac.jp
Gastroenterological reflux disease and functional dyspepsia are usually treatable using Western medical practices. Nonetheless, some cases present with intractable symptoms that are not amenable to these therapies. Treatment with kampo, a traditional Japanese medicine, recently has been proposed as an alternative therapy for use in combination with the Western practices. In general, traditional Japanese medicines have been used empirically for intractable symptoms correctively designated as "general malaises." Accumulating lines of evidence, including basic and clinical researches, have demonstrate detailed mechanisms where traditional Japanese medicines exert pharmacological action to improve symptoms. Therefore, traditional Japanese medicines have been gaining use by various medical doctors as the specific modes of pharmacological action are recognized. This review covers both the pharmacological functions and the clinical efficacies of rikkunshito for use in treating disorders of the gastrointestinal tract.

Keywords: general malaises, gastroesophageal reflux diseases, functional dyspepsia, gastrointestinal motility, acotiamide

\section{INTRODUCTION}

In Japan, an education in the practice of traditional Japanese medicines was not popular in the standard educational system until recently. This circumstance may be due to the fact that the detailed mechanism(s) of the pharmacological action of traditional Japanese medicines, including rikunshito, remains unknown. Such mechanism(s) have been difficult to define, in part because traditional Japanese medicines usually incorporate a variety of components. As a result, many traditional medicines have been used empirically that is, for the treatment of specific symptom. For instance, the traditional Japanese medicine, kampo, often is prescribed (typically as combination therapy with modern Western practices) for patients with medically unexplained physical symptoms or intractable gastrointestinal (GI) symptoms. This patient group represents a distinct population compared to that of patients with more typical and milder symptoms. Therefore kampo therapy, like many traditional Japanese medicines falls within the field of experience-based medicine, in contrast to that of evidence-based medicine. However, evidence for the efficacy of traditional Japanese medicines, particularly for rikkunshito, continues to accumulate (Tominaga and Arakawa, 2013). Hence, this review covers both basic and clinical evidences of rikkunshito, including pharmacological functions and clinical efficacies for the treatment and management of disorders of the GI tract.

\section{WHAT IS RIKKUNSHITO?}

Rikkunshito first appeared in the Japanese literature in the 1500s. This agent has since become one of most famous and prescribed medicines among the traditional medicines in Japan. Rikkunshito is composed of 8 constituents: Glycyrrhizae radix, Zingiberis rhizoma, Atractylodis lanceae rhizoma, Zizyphi fructus, Aurantii nobilis pericarpium, Ginseng radix, Pinelliae tuber, and Hoelen (Hattori et al., 2010). In recent times, analysis of the components of rikkunshito has been actively performed using high performance liquid chromatography. This work revealed that rikkunshito contains several ingredients such as liquiritin apioside, liquiritin, liquiritigenin, isoliquiritin apioside, isoliquiritin, isoliquiritigenin, glycyrrhizin, narirutin, and hesperidin (Endo et al., 2014; Figure 1). Recently, hesperidin was shown to have the highest potency (among these components) in a model of gastric emptying delay. Together, these findings support the broad use of rikkunshito for patients with dysfunction of the upper GI tract, including abdominal bloating, discomfort, nausea, and anorexia.

\section{BASIC PHARMACOLOGICAL FUNCTION OF RIKKUNSHITO: PROTECTIVE EFFECT ON MUCOSAL INJURIES AND PROKINETIC EFFECT ON GI MOTILITY}

Gastric mucosa is sometimes injured by various factors such as ethanol, non-steroidal anti-inflammatory drugs, systemic stress, or Helicobacter pylori infection (Watanabe et al., 2000; Hamaguchi et al., 2001; Takeuchi, 2012). Proton pump inhibitors (PPIs), histamine type 2 blockers, or mucoprotective agents are effective for treating such acute mucosal injuries (Arakawa et al., 1998; Yanaka et al., 2007). Thus, Western medical practices are sufficient for the treatment of such conditions. In addition to these conventional drugs, traditional Japanese medicines, including rikkunshito, also 


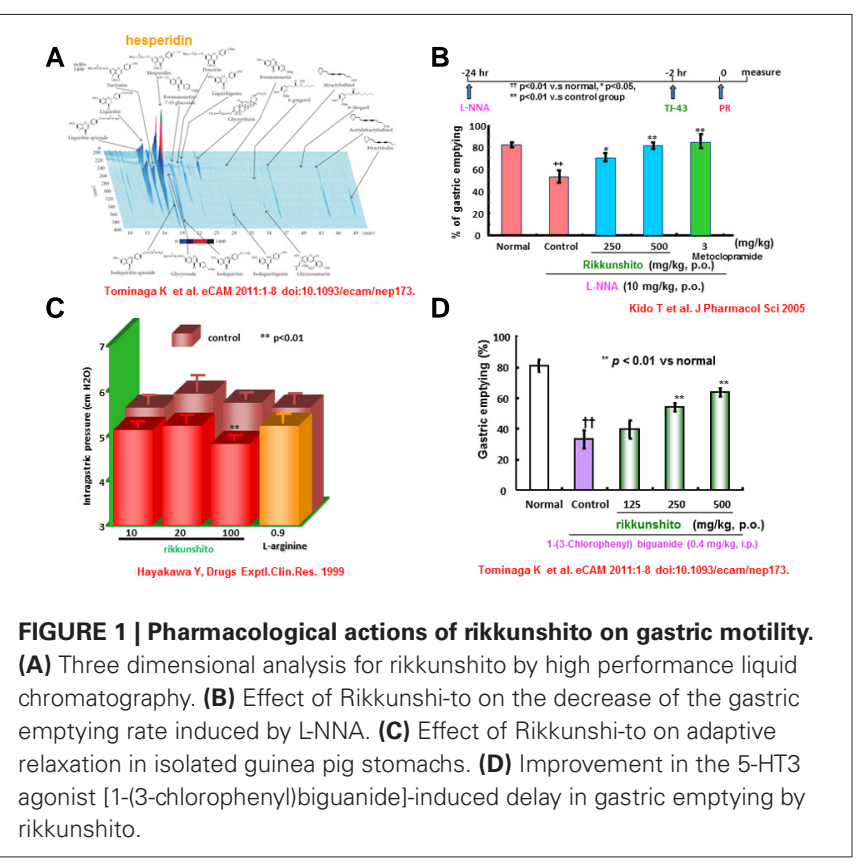

can protect against acute gastric mucosal injuries. Traditional Japanese medicines, including rikkunshito, are equality effective in treating ethanol-induced acute gastric mucosal injuries, through the amelioration of mucin content decrease of the gastric mucosa (Goso et al., 1996). Such effect is also partially mediated by nitric oxide (NO) but not by prostaglandins or sulfhydryls (Arakawa et al., 1999). Therefore, rikkunshito exerts protective effect against acute mucosal injuries in the rat model. However, there is no definitive evidence for this pharmacological function of rikkunshito under the acute and chronic conditions of the human stomach.

Gastric motility, a physiological function of the GI tract, generally consists of three phases: reservoir, churning, and emptying. The total coordination of these three phases is considered essential to healthy GI physiology. Among the three phases needed for gastric motility, gastric accommodation is considered to be the most important. Gastric accommodation provides the reservoir function, which originates in the proximal stomach and requires the activity of the neuronal transmitter NO (Desai et al., 1991; Uno et al., 1997). Rikkunshito is known to promote gastric accommodation in isolated guinea pig stomach (Hayakawa et al., 1999). Rikkunshito also relaxes the smooth muscles at the fundus portion of stomach isolated from diabetic neuropathic rats with gastric dysmotility (Kito and Suzuki, 2010). The observed pharmacological functions also are induced by L-arginine, a substrate of NO synthase that is also a component of rikkunshito. Like prokinetic agents, rikkunshito counteracts the attenuation of gastric dysmotility resulting from inhibition of $\mathrm{NO}$ synthesis (Hayakawa et al., 1999; Kido et al., 2005). Furthermore, rikkunshito attenuates the delay of gastric emptying mediated by the serotonin (5-HT) type 3 receptor, but does not counteract the delay caused by activation of dopamine receptors (Tominaga et al., 2009). Together, these findings indicate that rikkunshito can ameliorate gastric dysmotility mediated by $\mathrm{NO}$ or 5-HT pathways (Kido et al., 2005; Tominaga et al., 2009). Interestingly, rikkunshito enhances endogenous ghrelin levels in plasma, and regulates esophageal and gastric motilities (Takeda et al., 2008; Yakabi et al., 2010). Ghrelin is known to have a strong orexigenic effect (Kojima et al., 1999; Nakazato et al., 2001) and enhance GI motility (Fujino et al., 2003; Inui et al., 2004; Yang et al., 2014). It also has reported that rikkunshito attenuates impairment of GI motility via dysfunction of ghrelin signaling in a rat model of gastroesophageal reflux disease (GERD; Nahata et al., 2012). More recently, Nahata et al. (2014) extended this work to a model of gastric dysmotility. Those authors reported that acute restraint stress caused plasma acylated/desacyl ghrelin imbalance and gastric dysmotility in rat, and that exogenously administered acylated ghrelin relieved the gastric dysfunction caused by this stress. Rikkunshito was shown to enhance endogenous ghrelin signaling and to provide similar relief of stress-induced gastric dysmotility (Nahata et al., 2014). In a model of reflux esophagitis (RE), rikkunshito also inhibited the activation of ERK1/2 and decreased substance $\mathrm{P}$ and calcitonin gene-related peptide (CGRP) levels in Th8-10 dorsal root ganglia (Kondo et al., 2014). This finding may indicate that rikkunshito inhibits afferent neuronal activity associated with visceral pain, ameliorating decreased voluntary movement in this model. Together, these research data suggest that rikkunshito may be a promising drug for the treatment of GERD and functional dyspepsia (FD) in the clinic; this medicine's activity presumably is mediated by its pharmacological effects on esophageal and gastric motilities (Figure 1).

\section{CLINICAL EFFICACIES OF RIKKUNSHITO FOR UPPER GI SYMPTOMS \\ TREATMENT WITH RIKKUNSHITO FOR GERD}

The prevalence of obesity and metabolic syndrome in Japan continues to rise. At the same time, the rate of $H$. pylori infection in Japan is on the decrease. Under these circumstances, the prevalence of GERD is expected to increase in Japan, as has been seen in Western countries (Fujiwara and Arakawa, 2009; Fujikawa et al., 2012). The reflux of gastric contents, consisting primarily of gastric acid, is reported to be principal source of the pathogenesis of GERD (Vakil et al., 2006). Additionally, transient lower esophageal sphincter relaxation (TLESR) also is recognized to be critical to the pathogenesis of GERD (Hershcovici et al., 2011). Further GERD-associated factors include esophageal dysmotility, hypersensitivity to gastric acid or bile acid within the lower part of the esophagus, and gastric dysmotility affecting gastric accommodation and emptying. PPIs have been established as among the most effective drugs for treatment of GERD (Coté and Howden, 2008). However, other drugs regulating the esophageal motor functions also may prove useful in GERD treatment. Based on the above-described data on rikkunshito, the agent is a potential candidate treatment for patients with GERD, and so has been the subjects of clinical research. In a study that used $\mathrm{pH}$ multichannel intraluminal impedance monitoring, rikkunshito increased esophageal clearance and decreased esophageal acid exposure time, yielding relief of nausea symptoms in children with GERD (Kawahara et al., 2014). This traditional medicine also has been reported to relieve heartburn and acid regurgitation (Hiyama et al., 2008). 
In our own clinical work, we often encounter adult patients with PPI-refractory GERD, although PPIs typically are very effective for treating this disease. Anecdotally, PPI-refractory GERD often is observed in patients lacking erosions of the esophageal mucosa (non-erosive reflux disease, NERD). In the GERD 4 study (a randomized, parallel comparative study of PPI-refractory patients with GERD), combined treatment with rikkunshito and standard-dose rabeprazole showed an efficacy similar to that of double-dose PPI treatment (Tominaga et al., 2012). In a subsequent study (G-PRIDE: A randomized, placebo-controlled, double-blind clinical trial of rikkunshito for patients with NERD refractory to PPI), rikkunshito improved psychological quality of life (QOL) compared to the placebo control (Tominaga et al., 2014). In addition, among secondary endpoints, significant efficacy of rikkunshito was observed for acid-related dyspeptic symptoms, especially among non-obese patients, women, and the elderly. Subclass analysis of the elderly PPI-refractory NERD patients in the G-PRIDE study also showed that rikkunshito significantly improved total and acid-related dysmotility scores after the 8-week treatment interval compared to placebo. In addition, 8-week combination therapy with rikkunshito significantly improved symptoms of abdominal bloating, "heaviness" of the stomach, sick feeling after meals, and heartburn after meals (Sakata et al., 2014). Beyond standard symptoms, patients with GERD also are known to exhibit extra-esophageal symptoms. Interestingly, rikkunshito has been shown to be effective for patients with globus sensation, an effect mediated by attenuation of delayed gastric emptying (Tokashiki et al., 2013).

On the other hand, rikkunshito also has activities distinct from prokinetic functions. In a rat RE model, rikkunshito was shown to promote tight junction protein formation and to contribute to the repair of the dilation of intercellular spaces in the epithelial mucosa (Miwa et al., 2010). As with gastric acid, bile acid reflux is important for mucosal hypersensitivity in the pathogenesis of PPI-refractory GERD. Rikkunshito is reported to exhibit potent and differential absorption of bile salts (Araki et al., 2012). Notably, these findings suggest that rikkunshito may attenuate mucosal hypersensitivity to gastric acid or bile acid. These distinct pharmacological functions of rikkunshito may be a mechanism for relief of GERD symptoms in PPI-refractory NERD patients. Currently, long-term maintenance and enhanced therapies are recommended as future therapeutic regimens for PPI-refractory GERD patients (Kinoshita et al., 2012). Based upon these evidences, treatment regiments incorporating rikkunshito may be beneficial to GERD patients, particularly for PPI-refractory cases. (Figure 2).

\section{TREATMENT WITH RIKKUNSHITO FOR FD}

Functional dyspepsia presents as symptomatic gastritis. Pathogenesis of this disease is similar to that of chronic gastritis associated with $H$. pylori infection. Therefore, FD recently has been categorized as a disease distinct from chronic gastritis, and therefore has been classified as a member of the functional gastrointestinal disorders (FGIDs). According to the 2006 Rome III criteria (Drossman, 2006), the diagnosis of FGIDs is indicated in FD patients exhibiting any of four symptoms (epigastric pain, epigastric burning, postprandial fullness, or early satiety) orig-

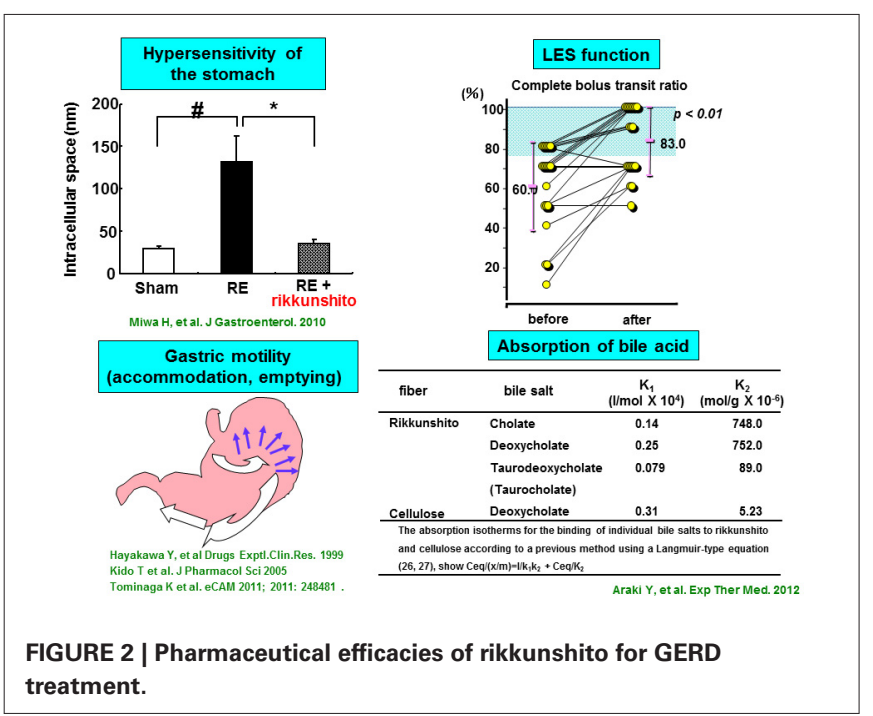

inating from the gastroduodenal tract without evident organic injuries. These symptoms often persist even after a variety of treatments, decreasing the QOL in patients with FD (Kinoshita et al., 2011). The pathophysiology of FD can reflect disorders of GI motility (Tominaga et al., 2008), acid secretion (el-Omar et al., 1995; Iwakiri et al., 2013), visceral hypersensitivity (Farré et al., 2013), H. pylori infection (Mönnikes et al., 2005), psychological factors (Tominaga et al., 2007; Ochi et al., 2008), and imbalance of the autonomic nervous system (ANS; Park et al., 2001). Of these factors, GI dysmotility has been most frequently associated with the pathogenesis of FD; however, this emphasis may reflect the relative ease of evaluating the physiological function of the GI tract in patients with FD. Gastric motility, notably its delay, has been a focus of evaluation in the pathogenesis of FD (Brun and Kuo, 2010). However, total coordination of gastric motility needs to be further elucidated for its potential role in the pathogenesis of FD. Indeed, disorder of gastric accommodation may cause epigastric discomfort, early satiety, and bloating in patients with FD (Bredenoord et al., 2003). Previously, we have reported that the impairment of gastric accommodation is associated with a delay in gastric emptying, resulting in dyspeptic symptoms (Tominaga et al., 2008). Recently, the high efficacy of acotiamide was demonstrated for treatment of dyspeptic symptoms in FD patients, an effect mediated by improvement of gastric accommodation (Matsueda et al., 2012; Kusunoki et al., 2012). Based on the results of various clinical trials, only acid suppressant drugs, prokinetic drugs, and eradication therapy for $H$. pylori have (until recently) been strongly recommended in Japan for treatment of FD. In this context, rikkunshito has been reported to ameliorate the delay in gastric emptying in patients with non-ulcer dyspepsia (NUD; Tatsuta and Iishi, 1993). The same report indicated that rikkunshito also alleviated various upper GI symptoms in patients with NUD (Tatsuta and Iishi, 1993). Moreover, rikkunshito has demonstrated efficacy for attenuation of impaired gastric accommodation and gastric motility (Kusunoki et al., 2010). Separate from stress' effects on gastric motility in the pathogenesis of FD, physical and psychological stresses often cause gastric hypersensitivity to stimulation by mechanical balloon distension. 
Stress-induced gastric hypersensitivity and/or changes in gastric wall tone (as assessed by gastric barostat method) were relieved by rikkunshito (Shiratori et al., 2011). In a separate human study comparing rikkunshito and domperidone, rikkunshito improved symptoms of patients with FD and increased plasma ghrelin levels (Arai et al., 2012). Rikkunshito was shown to ameliorate delayed gastric emptying in severely handicapped patients (Kawahara et al., 2009) and to improve gastric myoelectric activity in post-operative dyspeptic children after GI surgery (Yagi et al., 2004). These findings suggest that rikkunshito affects some inflammatory and/or neuroendocrinal mediators as well as gastric sensorimotor function, and improves dyspeptic symptoms of FD. Apart from FD, GI symptoms sometimes occur after endoscopic treatment. Combination therapy with rikkunshito and PPI after endoscopic submucosal dissection alleviated abdominal pain symptoms (Uehara et al., 2013). Recently, a randomized clinical trial using rikkunshito was performed for FD. Eightweek treatment with rikkunshito was tended to be effective for FD, as determined by global patient assessment (Suzuki et al., 2014). Thus, rikkunshito is a potential candidate for the clinical treatment of FD.

In conclusion, rikkunshtio is considered "complementary" (non-mainstream) medicine for treatment of GI diseases such as GERD and FD. However, rikkunshito frequently is prescribed for patients with diverse GI tract disorders. Combination treatments using modern Western and traditional Japanese medicines should be considered, especially in patients presenting with intractable symptioms, including general malaises.

\section{REFERENCES}

Arai, M., Matsumura, T., Tsuchiya, N., Sadakane, C., Inami, R., Suzuki, T., et al. (2012). Rikkunshito improves the symptoms in patients with functional dyspepsia, accompanied by an increase in the level of plasma ghrelin. Hepatogastroenterology 59, 62-66. doi: 10.5754/hge11246

Arakawa, T., Higuchi, K., Fujiwara, Y., Watanabe, T., Tominaga, K., Hayakawa, T., et al. (1999). Gastroprotection by Liu-Jun-Zi-Tang (TJ-43): possible mediation of nitric oxide but not prostaglandins or sulfhydryls. Drugs Exp. Clin. Res. 25, 207-210.

Arakawa, T., Kobayashi, K., Yoshikawa, T., and Tarnawski, A. (1998). Rebamipide: overview of its mechanisms of action and efficacy in mucosal protection and ulcer healing. Dig. Dis. Sci. 43, 5S-13S.

Araki, Y., Mukaisho, K. I., Fujiyama, Y., Hattori, T., and Sugihara, H. (2012). The herbal medicine rikkunshito exhibits strong and differential adsorption properties for bile salts. Exp. Ther. Med. 3, 645-649.

Bredenoord, A. J., Chial, H. J., Camilleri, M., Mullan, B. P., and Murray, J. A. (2003). Gastric accommodation and emptying in evaluation of patients with upper gastrointestinal symptoms Clin. Gastroenterol. Hepatol. 1, 264-272. doi: 10.1016/S1542-3565(03)00130-7

Brun, R., and Kuo, B. (2010). Functional dyspepsia. Therap. Adv. Gastroenterol. 3, 145-164. doi: 10.1177/1756283X10362639

Coté, G. A., and Howden, C. W. (2008). Potential adverse effects of proton pump inhibitors. Curr. Gastroenterol. Rep. 10, 208-214. doi: 10.1007/s11894-008-0045-4

Desai, K. M., Zembowicz, A., Sessa, W. C., and Vane, J. R. (1991). Nitroxergic nerves mediate vagally induced relaxation in the isolated stomach of the guinea pig. Proc. Natl. Acad. Sci. U.S.A. 88, 11490-11494.

Drossman, D. A. (2006). The functional gastrointestinal disorders and the Rome III process. Gastroenterology 130, 1377-1390. doi: 10.1053/j.gastro.2006.03.008

el-Omar, E., Penmann, I., Ardill, J. E., and McColl, K. E. (1995). A substantial proportion of non-ulcer dyspepsia patients have the same abnormality of acid secretion as duodenal ulcer patients. Gut 36, 534-538. doi: 10.1136/gut.36.4.534

Endo, M., Hori, M., Ozaki, H., Oikawa, T., and Hanawa, T. (2014). Rikkunshito, a Kampo medicine, ameliorates post-operative ileus by anti-inflammatory action. J. Pharmacol. Sci. 124, 374-385. doi: 10.1254/jphs.13182FP
Farré, R., Vanheel, H., Vanuytsel, T., Masaoka, T., Törnblom, H., Simrén, M., et al. (2013). In functional dyspepsia, hypersensitivity to postprandial distention correlates with meal-related symptom severity. Gastroenterology 145, 566-573. doi: 10.1053/j.gastro.2013.05.018

Fujikawa, Y., Tominaga, K., Fujii, H., Machida, H., Okazaki, H., Yamagami, H., et al. (2012). High prevalence of gastroesophageal reflux symptoms in patients with non-alcoholic fatty liver disease associated with serum levels of triglyceride and cholesterol but not simple visceral obesity. Digestion 86, 228-237. doi: $10.1159 / 000341418$

Fujino, K., Inui, A., Asakawa, A., Kihara, N., Fujimura, M., and Fujimiya, M. (2003). Ghrelin induces fasted motor activity of the gastrointestinal tract in conscious fed rats. J. Physiol. 550, 227-240. doi: 10.1113/jphysiol.2003.040600

Fujiwara, Y., and Arakawa, T. (2009). Epidemiology and clinical characteristics of GERD in the Japanese population. J. Gastroenterol. 44, 518-534. doi: 10.1007/s00535-009-0047-5

Goso, Y., Ogata, Y., Ishihara, K., and Hotta, K. (1996). Effects of traditional herbal medicine on gastric mucin against ethanol-induced gastric injury in rats. Comp. Biochem. Physiol. C Pharmacol. Toxicol. Endocrinol. 113, 17-21. doi: 10.1016/0742-8413(95)02042-X

Hamaguchi, M., Watanabe, T., Higuchi, K., Tominaga, K., Fujiwara, Y., and Arakawa, T. (2001). Mechanisms and roles of neutrophil infiltration in stressinduced gastric injury in rats. Dig. Dis. Sci. 46, 2708-2715. doi: 10.1023/ A:1012779530004

Hattori, T., Fujitsuka, N., Asakawa, A., and Inui, A. (2010). "A strategy using Rikkunshito (Liu-Jun-Zi-Tang), a Japanese traditional medicine, to treat gastrointestinal disease," in Basics of Evidences-Based Herbal Medicine, ed. H. Satou (Trivandrum: Research Signpost press), 149-60.

Hayakawa, T., Arakawa, T., Kase, Y., Akiyama, S., Ishige, A., Takeda, S., et al. (1999). Liu-Jun-Zi-Tang, a kampo medicine, promotes adaptive relaxation in isolated guinea pig stomachs. Drugs Exptl. Clin. Res. 25, 211-218.

Hershcovici, T., Mashimo, H., and Fass, R. (2011). The lower esophageal sphincter. Neurogastroenterol. Motil. 23, 819-830. doi: 10.1111/j.1365-2982.2011.01738.x

Hiyama, T., Yoshihara, M., Tanaka, S., Haruma, K., and Chayama, K. (2008). Strategy for treatment of non-erosive reflux disease in Asia. World J. Gastroenterol. 14, 3123-3128. doi: 10.3748/wjg.14.3123

Inui, A., Asakawa, A., Bowers, C. Y., Mantovani, G., Laviano, A., Meguid, M. M., et al. (2004). Ghrelin, appetite, and gastric motility: the emerging role of the stomach as an endocrine organ. FASEB J. 18, 439-456. doi: 10.1096/fj.03-0641 rev

Iwakiri, R., Tominaga, K., Furuta, K., Inamori, M., Furuta, T., Masuyama, H., et al. (2013). Randomised clinical trial: rabeprazole improves symptoms in patients with functional dyspepsia in Japan. Aliment. Pharmacol. Ther. 38, 729-740. doi: 10.1111/apt.12444

Kawahara, H., Mitani, Y., Nomura, M., Nose, K., Yoneda, A., Hasegawa, T., et al. (2009). Impact of rikkunshito, an herbal medicine, on delayed gastric emptying in profoundly handicapped patients. Pediatr. Surg. Int. 25, 987-990. doi: 10.1007/s00383-009-2453-4

Kawahara, H., Tazuke, Y., Soh, H., Yoneda, A., and Fukuzawa, M. (2014). Physiological analysis of the effects of rikkunshito on acid and non-acid gastroesophageal reflux using $\mathrm{pH}$-multichannel intraluminal impedance monitoring. Pediatr. Surg. Int. 30, 927-931. doi: 10.1007/s00383-014-3565-Z

Kido, T., Nakai, Y., Kase, Y., Sakakibara, I., Nomura, M., Takeda, S., et al. (2005). Effects of rikkunshi-to, a traditional Japanese medicine, on the delay of gastric emptying induced by N(G)-nitro-L-arginine. J. Pharmacol. Sci. 98, 161-167. doi: 10.1254/jphs.FPJ04056X

Kinoshita, Y., Chiba, T., and FUTURE study group. (2011). Therapeutic effects of famotidine on chronic symptomatic gastritis: subgroup analysis from FUTURE study. J. Gastroenterol. 47, 377-386. doi: 10.1007/s00535-011-0503-x

Kinoshita, Y., Hongo, M., and Japan TWICE Study Group. (2012). Efficacy of twicedaily rabeprazole for reflux esophagitis patients refractory to standard oncedaily administration of PPI: the Japan-based TWICE study. Am. J. Gastroenterol. 107, 522-530. doi: 10.1038/ajg.2012.19

Kito, Y., and Suzuki, H. (2010). Properties of Rikkunshi-to (TJ-43)-induced relaxation of rat gastric fundus smooth muscles. Am. J. Physiol. Gastrointest. Liver Physiol. 298, G755-G763. doi: 10.1152/ajpgi.00333.2009

Kojima, M., Hosoda, H., Date, Y., Nakazato, M., Matsuo, H., and Kangawa, K. (1999). Ghrelin is a growth-hormone-releasing acylated peptide from stomach. Nature 402, 656-660. doi: 10.1038/45230

Kondo, T., Oshima, T., Koseki, J., Hattori, T., Kase, Y., Tomita, T., et al. (2014). Effect of rikkunshito on the expression of substance $\mathrm{P}$ and CGRP in dorsal root 
ganglion neurons and voluntary movement in rats with experimental reflux esophagitis. Neurogastroenterol. Motil. 26, 913-921. doi: 10.1111/nmo.12342

Kusunoki, H., Haruma, K., Hata, J., Ishii, M., Kamada, T., Yamashita, N., et al. (2010). Efficacy of Rikkunshito, a traditional Japanese medicine (Kampo), in treating functional dyspepsia. Intern. Med. 49, 2195-2202. doi: 10.2169/internalmedicine.49.3803

Kusunoki, H., Haruma, K., Manabe, N., Imamura, H., Kamada, T., Shiotani, A, et al. (2012). Therapeutic efficacy of acotiamide in patients with functional dyspepsia based on enhanced postprandial gastric accommodation and emptying: randomized controlled study evaluation by real-time ultrasonography. Neurogastroenterol. Motil. 24, 540-545, e250-e251. doi: 10.1111/j.1365-2982. 2012.01897.x

Matsueda, K., Hongo, M., Tack, J., Saito, Y., and Kato, H. (2012). A placebocontrolled trial of acotiamide for meal-related symptoms of functional dyspepsia. Gut 61, 821-828. doi: 10.1136/gutjnl-2011-301454

Miwa, H., Koseki, J., Oshima, T., Kondo, T., Tomita, T., Watari, J., et al. (2010). Rikkunshito, a traditional Japanese medicine, may relieve abdominal symptoms in rats with experimental esophagitis by improving the barrier function of epithelial cells in esophageal mucosa. J. Gastroenterol. 45, 478-487. doi: 10.1007/ s00535-009-0180-1

Mönnikes, H., van der Voort, I. R., Wollenberg, B., Heymann-Monnikes, I., Tebbe, J. J., Alt, W., et al. (2005). Gastric perception thresholds are low and sensory neuropeptide levels high in Helicobacter pylori-positive functional dyspepsia. Digestion 71, 111-123. doi: 10.1159/000084625

Nahata, M., Muto, S., Oridate, N., Ohnishi, S., Nakagawa, K., Sadakane, C., et al. (2012). Impaired ghrelin signaling is associated with gastrointestinal dysmotility in rats with gastroesophageal reflux disease. Am. J. Physiol. Gastrointest. Liver Physiol. 303, G42-G53. doi: 10.1152/ajpgi.00462.2011

Nahata, M., Saegusa, Y., Sadakane, C., Yamada, C., Nakagawa, K., Okubo, N., et al. (2014). Administration of exogenous acylated ghrelin or rikkunshito, an endogenous ghrelin enhancer, improves the decrease in postprandial gastric motility in an acute restraint stress mouse model. Neurogastroenterol. Motil. 6, 821-831. doi: 10.1111/nmo.12336

Nakazato, M., Murakami, N., Date, Y., Kojima, M., Matsuo, H., Kangawa, K., et al. (2001). A role for ghrelin in the central regulation of feeding. Nature 409, 194198. doi: $10.1038 / 35051587$

Ochi, M., Tominaga, K., Iketani, T., Kadouchi, K., Tanigawa, T., Shiba, M., et al. (2008). Perfectionism underlying psychological background correlated with the symptoms of functional dyspepsia. J. Gastroenterol. 43, 699-704. doi: 10.1007/ s00535-008-2210-9

Park, D. I., Rhee, P. L., Kim, Y. H., Sung, I. K., Son, H. J., Kim, J. J., et al. (2001). Role of autonomic dysfunction in patients in patients with functional dyspepsia. Dig. Liver Dis. 33, 464-471. doi: 10.1016/S1590-8658(01)80023-2

Sakata, Y., Tominaga, K., Kato, M., Takeda, H., Shimoyama, Y., Takeuchi, T., et al. (2014). Clinical characteristics of elderly patients with proton pump inhibitorrefractory non-erosive reflux disease from the G-PRIDE study who responded to rikkunshito. BMC Gastroenterol. 14:116. doi: 10.1186/1471-230X-14-116

Shiratori, M., Shoji, T., Kanazawa, M., Hongo, M., and Fukudo, S. (2011). Effect of rikkunshito on gastric sensorimotor function under distention. Neurogastroenterol. Motil. 23, 323-329. e155-e156. doi: 10.1111/j.1365-2982.2010.01648.x

Suzuki, H., Matsuzaki, J., Fukushima, Y., Suzaki, F., Kasugai, K., Nishizawa, T., et al. (2014). Rikkunshito study group. Randomized clinical trial: rikkunshito in the treatment of functional dyspepsia-a multicenter, double-blind, randomized, placebo-controlled study. Neurogastroenterol. Motil. 26, 950-9561. doi: 10.1111/nmo. 12348

Takeda, H., Sadakane, C., Hattori, T., Katsurada, T., Ohkawara, T., Nagai, K., et al. (2008). Rikkunshito, an herbal medicine, suppresses cisplatin-induced anorexia in rats via 5-HT2 receptor antagonism. Gastroenterology 134, 2004-2013. doi: 10.1053/j.gastro.2008.02.078

Takeuchi, K. (2012). Pathogenesis of NSAID-induced gastric damage: importance of cyclooxygenase inhibition and gastric hypermotility. World J. Gastroenterol. 18, 2147-2160. doi: 10.3748/wjg.v18.i18.2147

Tatsuta, M., and Iishi, H. (1993). Effect of treatment with Lui-Jun-Zi-Tang (TJ-43) on gastric emptying and gastrointestinal symptoms in dyspeptic patients. Aliment. Pharmacol. Ther. 7, 459-462. doi: 10.1111/j.1365-2036.1993.tb00120.x

Tokashiki, R., Okamoto, I., Funato, N., and Suzuki, M. (2013). Rikkunshito improves globus sensation in patients with proton-pump inhibitor-refractory laryngopharyngeal reflux. World J. Gastroenterol. 19, 5118-5124. doi: 10.3748/ wjg.v19.i31.5118
Tominaga, K., and Arakawa, T. (2013). Kampo medicines for gastrointestinal tract disorders: a review of basic science and clinical evidence and their future application. J. Gastroenterol. 48, 452-462. doi: 10.1007/s00535-013-0788-z

Tominaga, K., Higuchi, K., Iketani, T., Ochi, M., Kadouchi, K., Tanigawa, T., et al. (2007). Comparison of gastrointestinal symptoms and psychological factors of functional dyspepsia to peptic ulcer or panic disorder patients. Inflammopharmacology 15, 84-89. doi: 10.1007/s10787-006-0011-4

Tominaga, K., Higuchi, K., Ochi, M., Kadouchi, K., Kawamura, E., Tanigawa, T., et al. (2008). Concurrent assessment of reservoir and emptying of the stomach for dyspepsia patients. Hepatogastroenterology 55, 744-749.

Tominaga, K., Iwakiri, R., Fujimoto, K., Fujiwara, Y., Tanaka, M., Shimoyama, Y., et al. (2012). Rikkunshito improves symptoms in PPI-refractory GERD patients: a prospective, randomized, multicenter trial in Japan. J. Gastroenterol. 47, 284292. doi: 10.1007/s00535-011-0488-5

Tominaga, K., Kato, M., Takeda, H., Shimoyama, Y., Umegaki, E., Iwakiri, R., et al. (2014). A randomized, placebo-controlled, double-blind clinical trial of rikkunshito for patients with non-erosive reflux disease refractory to proton-pump inhibitor: the G-PRIDE study. J. Gastroenterol. 49, 1392-1405. doi: 10.1007/ s00535-013-0896-9

Tominaga, K., Kido, T., Ochi, M., Sadakane, C., Mase, A., Okazaki, H., et al. (2009). The traditional Japanese medicine rikkunshito promotes gastric emptying via the antagonistic action of the 5-HT3 receptor pathway in rats. Evid. Based Complement. Alternat. Med. 2011: 248481. doi: 10.1093/ecam/nep173

Uehara, R., Isomoto, H., Minami, H., Yamaguchi, N., Ohnita, K., Ichikawa, T., et al. (2013). Characteristics of gastrointestinal symptoms and function following endoscopic submucosal dissection and treatment of the gastrointestinal symptoms using rikkunshito. Exp. Ther. Med. 6, 1083-1088.

Uno, H., Arakawa, T., Fukuda, T., Higuchi, K., and Kobayashi, K. (1997). Involvement of capsaicin-sensitive sensory nerves in gastric adaptive relaxation in isolated guinea-pig stomachs. Digestion 58, 232-239. doi: 10.1159/000201449

Vakil, N., van Zanten, S. V., Kahrialis, P., Dent, J., Jones, R., and Global Consensus Group. (2006). The Montreal definition and classification of gastroesophageal reflux disease: a global evidence-based consensus. Am. J. Gastroenterol. 101, 1900-1920. doi: 10.1111/j.1572-0241.2006.00630.x

Watanabe, T., Higuchi, K., Tominaga, K., Fujiwara, Y., and Arakawa, T. (2000). Cytoprotective effect of rabeprazole against ethanol-induced gastric mucosal damage: possible involvement of nitric oxide. Drugs Exp. Clin. Res. 26, 41-45.

Yagi, M., Homma, S., Kubota, M., Iinuma, Y., Kanada, S., Kinoshita, Y., et al. (2004). The herbal medicine Rikkunshi-to stimulates and coordinates the gastric myoelectric activity in post-operative dyspeptic children after gastrointestinal surgery. Pediatr. Surg. Int. 19, 760-765. doi: 10.1007/s00383-003-1053-y

Yakabi, K., Sadakane, C., Noguchi, M., Ohno, S., Ro, S., Chinen, K., et al. (2010). Reduced ghrelin secretion in the hypothalamus of rats due to cisplatin-induced anorexia. Endocrinology 151, 3773-3782. doi: 10.1210/en.2010-0061

Yanaka, A., Zhang, S., Sato, D., Tauchi, M., Suzuki, H., Shibahara, T., et al. (2007). Geranylgeranylacetone protects the human gastric mucosa from diclofenacinduced injury via induction of heat shock protein 70 . Digestion $75,148-155$. doi: $10.1159 / 000106756$

Yang, C. G., Liao, Z. F., Qiu, W. C., Yan, J., and Wang, Z. G. (2014). Function of ghrelin and ghrelin receptors in the network regulation of gastric motility. Mol. Med. Rep. 10, 2453-2458. doi: 10.3892/mmr.2014.2571

Conflict of Interest Statement: The authors declare that the research was conducted in the absence of any commercial or financial relationships that could be construed as a potential conflict of interest.

Received: 26 November 2014; accepted: 10 January 2015; published online: 30 January 2015.

Citation: Tominaga K and Arakawa T (2015) Clinical application of kampo medicine (rikkunshito) for common and/or intractable symptoms of the gastrointestinal tract. Front. Pharmacol. 6:7. doi: 10.3389/fphar.2015.00007

This article was submitted to Ethnopharmacology, a section of the journal Frontiers in Pharmacology.

Copyright $\odot 2015$ Tominaga and Arakawa. This is an open-access article distributed under the terms of the Creative Commons Attribution License (CC BY). The use, distribution or reproduction in other forums is permitted, provided the original author(s) or licensor are credited and that the original publication in this journal is cited, in accordance with accepted academic practice. No use, distribution or reproduction is permitted which does not comply with these terms. 